\title{
Modeling Light Cold Dark Matter
}

\author{
Abdessamad Abada ${ }^{1}$ and Salah Nasri ${ }^{2}$ \\ ${ }^{1}$ Laboratoire de Physique des Particules et Physique Statistique, Ecole Normale Supérieure \\ ${ }^{2}$ Physics Department, UAE University, Al Ain \\ ${ }^{1}$ Algeria \\ ${ }^{2}$ United Arab Emirates
}

\section{Introduction}

Dark matter is known to contribute about $22 \%$ to the total mass density in the Universe. Its existence started to be noticed in 1933, when Fritz Zwicky made an estimate of the total mass of the Coma cluster of galaxies outside our local group (Zwicky, 1933). Assuming that the galaxies in that cluster form a gravitationally bound system, he measured the cluster's geometrical size and the velocity dispersion of galaxies in it via Doppler redshift. He found that the mass of the Coma cluster had to be about 400 times larger than the estimate based on the number of galaxies and the total brightness of the cluster. He concluded that there must be some 'non-visible' form of matter which would provide enough gravity to hold the cluster gravitationally bound. This non-visible mass is called 'dark matter'.

There is by now extensive astronomical evidence supporting the existence of dark matter. The strongest such evidence comes from the measurements of the circular velocity of stars and gas in spiral galaxies versus their radial distance. If one assumes that the bulb in the center of a typical spiral galaxy is spherically symmetric, then one would expect the orbital velocity $v(r)$ outside the disk to behave like $1 / \sqrt{r}$. Instead, the study of thousands of rotation curves of spiral galaxies shows that the orbital velocity rises from the center until it reaches a limiting value $v_{C} \sim(100-200) \mathrm{km} / \mathrm{s}$, and then stays flat outside the galaxy core (Persic \& Salucci \& Stel, 1996). For example, the observed velocity of the rotation curve of the spiral galaxy M33, one of the brightest spiral galaxies in our local group, at $r \simeq 10 \mathrm{kpc}$ is $v_{C} \simeq 120 \mathrm{~km} / \mathrm{s}$, whereas the expected velocity is $v \simeq 40 \mathrm{~km} / \mathrm{s}$. One infers from this that the total mass in the galaxy is about nine times the luminous matter $\left(\Omega_{\text {lum }} \sim 10 \%\right)$. This implies that there is about ten times more mass in the halo of spiral galaxies than in the disk.

There is also evidence of dark matter in elliptic galaxies and cluster of galaxies. This comes from the observation of $X$-rays emitted via the bremsstrahlung process $e+p \rightarrow e+p+\gamma$ from the intergalactic gas in the cluster. Assuming hydrostatic equilibrium, we can deduce from the measurement of the $X$-ray luminosity and the shape of its spectrum, assumed isothermal, the mass distribution in the galaxy that is necessary to bind the hot gas. The observations indicate that the total mass associated with these systems is considerably larger than the luminous component (Fabricant \& Gorenstein, 1983; Stewart et al., 1984). Note that cluster masses can also be determined from their lensing effect on light from distant sources (Mellier, 1999).

Furthermore, during the past few years, data from the WMAP satellite has provided us with the most precise measurements yet of the cosmological parameters (Spergel et al., 2007; Pope et al., 2004). By analyzing the location and the height of the acoustic peaks of the 
temperature fluctuations, one can extract the contribution of the different species to the critical energy density of the Universe. For instance, the height of the first peak relative to the second one gives a baryon density of about $4 \%$, which is consistent with the predictions of the primordial theory of Big Bang nucleosynthesis (Steigman, 2010). The third peak is sensitive to the amount of total matter density in the Universe and can be used to extract the energy density $\Omega_{\mathrm{DM}}$ of dark matter in the Universe. The best fit is (Komatsu, 2010):

$$
\Omega_{\mathrm{DM}} \bar{h}^{2}=0.1123 \pm 0.0035,
$$

where $\bar{h}$ is the Hubble constant in units of $100 \mathrm{~km} \times \mathrm{s}^{-1} \times \mathrm{Mpc}^{-1}$.

Yet, even though dark matter dominates the matter mass 'budget' of the Universe, its very nature remains elusive. Indeed, what are its quantum numbers, its mass? How does it interact with the Standard Model particles? One should also say that up until now, it has not been directly detected. But there is a number of basic properties that any candidate for dark matter should have ${ }^{1}$. First of all, it must be massive, and this is because of the non-relativistic velocities involved. Second, it must be stable so that it would survive until today, which means it must have a lifetime larger than that of the Universe. Third, it must be electrically neutral, otherwise it would have been very likely seen via its electromagnetic interaction with visible matter. Also, the abundance of such stable charged massive particles would be severely constrained, in particular from searches in the deep sea water (Amsler et al., 2008). Fourth, a dark-matter candidate should not interact strongly. Indeed, if such a massive stable particle could do so, it would be able to bind and form anomalously heavy nuclei. But the resulting number of such anomalously heavy nuclei that would be present today is shown to be excluded by existing searches (Javorsek, 2001; 2002). Fifth, for a dark matter candidate to act as a seed for structure formation, it must decouple at a temperature of the order of its mass. Such a candidate is known as "cold dark matter". Sixth, it must give the right relic dark-matter density, which, by the latest astrophysical observations, is about $22 \%$ of the total energy density in the Universe (Komatsu, 2010).

While the Standard Model of elementary particle Physics is very successful at describing the interactions between 'visible' particles, it cannot accommodate for a weakly interacting massive particle (WIMP) as a suitable candidate for dark matter. Hence, extensions of the Standard Model are inevitable and, given the elusiveness of dark matter, modeling becomes a necessity. In this framework, the most popular candidate for dark matter is the neutralino, a neutral $R$-odd supersymmetric particle. Indeed, neutralinos are produced or destroyed in pairs only, thus rendering the lightest SUSY particle (LSP) stable (Ellis et al., 1984). In the minimal version of the supersymmetric extension of the Standard Model, the neutralino $\chi_{1}^{0}$ is a linear combination of the fermionic partners of the neutral electroweak gauge bosons (gauginos) and the neutral Higgs bosons (higgsinos). It can annihilate through a t-channel sfermion exchange into Standard-Model fermions, or via a t-channel chargino-mediated process into $W^{+} W^{-}$, or through an s-channel pseudoscalar Higgs exchange into fermion pairs. Also, it can undergo elastic scattering with nuclei through mainly a scalar Higgs exchange (Jungman, 1996).

However, having a neutralino as a candidate for light dark matter can be a real challenge. For example, in mSUGRA, the constraint from WMAP observations and the bound on the pseudo-scalar Higgs mass from LEP give neutralino mass $m_{\chi_{1}^{0}} \geq 50 \mathrm{GeV}$ (Belanger et al., 2009; Akrami et al., 2010). If one allows the gaugino masses $M_{1}$ and $M_{2}$ to be free parameters

\footnotetext{
${ }^{1}$ We implicitly mean a candidate from the realm of elementary particles.
} 
and the gluino mass to satisfy the universal condition at some grand unification scale, that is, $M_{3}=3 M_{2}$, then the LSP should be heavier than about $28 \mathrm{GeV}$ (Vasquez et al., 2010), see also (Feldman, 2010; Kuflik, 2010). A similar analysis is done in (Fornengo et al., 2011) with the gluino mass taken as a free parameter, and it is concluded that the lower limit on the neutralino mass varies between about $7 \mathrm{GeV}$ and $12 \mathrm{GeV}$, depending on the gluino mass and the degeneracy of the squarks. In the extension of the MSSM with an extra singlet chiral superfield (NMSSM), a model with 11 input parameters, it is found that a neutralino with a mass of the order of a few GeVs is possible, with a higher likelihood peaked at around $15 \mathrm{GeV}$ (Vasquez et al., 2010).

Therefore, with the aim of modeling dark matter that could be as light as a few GeVs and maybe lighter, and with no clear clue yet as to what the internal structure of the WIMP is, if any, a 'pedestrian' approach can be attractive. In this logic, the simplest of models is to extend the Standard Model with a real scalar field, the dark matter, a Standard-Model gauge singlet that interacts with visible particles via the Higgs field only. To ensure stability, it is endowed with a discrete $\mathbb{Z}_{2}$ symmetry that does not break spontaneously. Such a model can be seen as a low-energy remnant of some higher-energy physics waiting to be understood. In this cosmological setting, such an extension has first been proposed in (Silveira, 1985) and further studied in (McDonald, 1994) where the unbroken $\mathbb{Z}_{2}$ symmetry is extended to a global $\mathrm{U}(1)$ symmetry. A more extensive exploration of the model and its implications was done in (Burgess et al., 2011), specific implications on Higgs detection and LHC physics discussed in (Barger et al., 2008) and one-loop vacuum stability looked into and perturbativity bounds obtained in (Gonderinger et al., 2010). However, the work (He et al., 2009; Asano \& Kitano, 2010) considers this minimal extension too and uses constraints from the direct-detection experiments XENON10 (Angle et al., 2008) and CDMSII (Ahmed et al., 2009) to exclude dark matter masses smaller than 50,70 and $75 \mathrm{GeV}$ for Higgs masses equal to 120, 200 and $350 \mathrm{GeV}$ respectively. Furthermore, it was recently shown that the Fermi-LAT data on the isotropic diffuse gamma-ray emission can potentially exclude this one-singlet dark-matter model for masses as low as $6 \mathrm{GeV}$, assuming a NFW profile for the dark-matter distribution (Arina \& Tytgat, 2011).

So, in order to allow for light dark matter in this 'bottom-up' approach, the natural step forward is to add another real scalar field, endowed with a $\mathbb{Z}_{2}$ symmetry too, but one which is spontaneously broken so that new channels for dark matter annihilation are opened, increasing this way the annihilation cross-section, hence allowing smaller masses for the WIMP. This auxiliary field must also be a Standard-Model gauge singlet. The present chapter introduces this extension and presents some of its aspects. The aim is to use this example as a generic prototype in order to show how modeling of cold dark matter can be done and what are the main steps to follow. Most of the technical material used here is drawn from (Abada, 2011).

This chapter is organized as follows. After this introduction, we present the model in the next section. The spontaneous breaking of the electroweak and the additional $\mathbb{Z}_{2}$ symmetries is performed in the usual way and the physical modes as well as the physical parameters are explained. There is mixing between the physical new scalar field and the Higgs, and this is one of the quantities parametrizing the subsequent physics. We discuss in section three the imposition of the constraint from the dark matter relic density on the dark-matter annihilation cross-section and study its effects. Of course, as we will see, the space of parameters is quite large and cannot be covered in its entirety in any study of reasonable size. Representative values have to be selected and the behavior of the model, as well as its 
capabilities, are described accordingly. Though our main interest in this work is light dark matter, yet we allow the dark-matter mass to vary from $0.1 \mathrm{GeV}$ to $100 \mathrm{GeV}$, sometimes higher. We find that the model is rich enough to bear dark matter for most of these masses, including those in the very light sector. In section four, we determine the total cross section $\sigma_{\text {det }}$ for non-relativistic elastic scattering of dark matter off a nucleon target and compare it to the current direct-detection experimental bounds and projected sensitivity. For this, we choose the results of CDMSII (Ahmed et al., 2009) and XENON100 (April et al., 2010), as well as the projections of SuperCDMS (Schnee et al., 2005) and XENON1T (April et al., 2010). Here too we cannot cover all of the parameters' space nor are we going to give a detailed account of the behavior of $\sigma_{\text {det }}$ as a function of the dark matter mass, but general trends are mentioned. In section five, we show how low-energy particle phenomenology can constrain the various parameters of the model. We have space for only one typical example, namely, the decay of the $B_{s}$ meson into a pair of $\mu^{-} \mu^{+}$. Here, we take from the start light dark matter, with a mass in the range $0.1 \mathrm{GeV}-10 \mathrm{GeV}$. Finally, in the last section, we finish the chapter with a number of concluding remarks.

\section{A two-singlet extension to the Standard Model}

The Standard Model is extended by two real, scalar, and $\mathbb{Z}_{2}$-symmetric fields. One is the dark matter field $S_{0}$ for which the $\mathbb{Z}_{2}$ symmetry is unbroken while the other field $\chi_{1}$ undergoes spontaneous symmetry breaking. Both fields are Standard-Model gauge singlets and hence, can interact with the other sectors of the Standard Model only via the Higgs doublet $H$. This latter is taken in the unitary gauge such that $H^{\dagger}=1 / \sqrt{2}\left(\begin{array}{ll}0 & h^{\prime}\end{array}\right)$, where $h^{\prime}$ is a real scalar. The potential function involving $S_{0}, h^{\prime}$ and $\chi_{1}$ is given by the following expression:

$$
U=\frac{\tilde{m}_{0}^{2}}{2} S_{0}^{2}-\frac{\mu^{2}}{2} h^{\prime 2}-\frac{\mu_{1}^{2}}{2} \chi_{1}^{2}+\frac{\eta_{0}}{24} S_{0}^{4}+\frac{\lambda}{24} h^{\prime 4}+\frac{\eta_{1}}{24} \chi_{1}^{4}+\frac{\lambda_{0}}{4} S_{0}^{2} h^{\prime 2}+\frac{\eta_{01}}{4} S_{0}^{2} \chi_{1}^{2}+\frac{\lambda_{1}}{4} h^{\prime 2} \chi_{1}^{2},
$$

where the mass-squared parameters $\tilde{m}_{0}^{2}, \mu^{2}$ and $\mu_{1}^{2}$ and all the coupling constants are real positive numbers. The Higgs field undergoes spontaneous electroweak symmetry breaking and oscillates around the vacuum expectation value $v=246 \mathrm{GeV}$ (Nakamura et al., 2010). The field $\chi_{1}$ will oscillate around the vacuum expectation value $v_{1}>0$. Both $v$ and $v_{1}$ are related to the parameters of the theory by the two relations:

$$
v^{2}=6 \frac{\mu^{2} \eta_{1}-6 \mu_{1}^{2} \lambda_{1}}{\lambda \eta_{1}-36 \lambda_{1}^{2}} ; \quad v_{1}^{2}=6 \frac{\mu_{1}^{2} \lambda-6 \mu^{2} \lambda_{1}}{\lambda \eta_{1}-36 \lambda_{1}^{2}} .
$$

The self-coupling constants are assumed sufficiently larger than the mutual ones and perturbation theory is assumed applicable throughout.

Writing $h^{\prime}=v+\tilde{h}$ and $\chi_{1}=v_{1}+\tilde{S}_{1}$, the potential function becomes, up to an irrelevant zero-field energy:

$$
U=U_{\text {quad }}+U_{\text {cub }}+U_{\text {quar }}
$$

where the mass-squared (quadratic) terms are gathered in $U_{\text {quad }}$, the cubic interactions in $U_{\text {cub }}$ and the quartic ones in $U_{\text {quar. }}$ The quadratic terms are given by:

$$
U_{\text {quad }}=\frac{1}{2} m_{0}^{2} S_{0}^{2}+\frac{1}{2} M_{h}^{2} \tilde{h}^{2}+\frac{1}{2} M_{1}^{2} \tilde{S}_{1}^{2}+M_{1 h}^{2} \tilde{h} \tilde{S}_{1},
$$


where the mass-squared coefficients are related to the original parameters of the theory by the following relations:

$$
\begin{aligned}
& m_{0}^{2}=\tilde{m}_{0}^{2}+\frac{\lambda_{0}}{2} v^{2}+\frac{\eta_{01}}{2} v_{1}^{2} ; \quad M_{h}^{2}=-\mu^{2}+\frac{\lambda}{2} v^{2}+\frac{\lambda_{1}}{2} v_{1}^{2} ; \\
& M_{1}^{2}=-\mu_{1}^{2}+\frac{\lambda_{1}}{2} v^{2}+\frac{\eta_{1}}{2} v_{1}^{2} ; \quad M_{1 h}^{2}=\lambda_{1} v v_{1} .
\end{aligned}
$$

As we see, in this basis, the mass-squared matrix is not diagonal: there is mixing between the fields $\tilde{h}$ and $\tilde{S}_{1}$. Denoting by $h$ and $S_{1}$ the physical field eigenmodes of the mass-squared matrix, we rewrite:

$$
U_{\text {quad }}=\frac{1}{2} m_{0}^{2} S_{0}^{2}+\frac{1}{2} m_{h}^{2} h^{2}+\frac{1}{2} m_{1}^{2} S_{1}^{2},
$$

where the physical fields are related to the mixed ones by a $2 \times 2$ rotation:

$$
\left(\begin{array}{c}
h \\
S_{1}
\end{array}\right)=\left(\begin{array}{cc}
\cos \theta & \sin \theta \\
-\sin \theta & \cos \theta
\end{array}\right)\left(\begin{array}{c}
\tilde{h} \\
\tilde{S}_{1}
\end{array}\right) \text {. }
$$

Here $\theta$ is the mixing angle, related to the original mass-squared parameters by the relation:

$$
\tan 2 \theta=\frac{2 M_{1 h}^{2}}{M_{1}^{2}-M_{h}^{2}}
$$

and the physical masses in (7) by the two relations:

$$
\begin{aligned}
& m_{h}^{2}=\frac{1}{2}\left[M_{h}^{2}+M_{1}^{2}+\varepsilon\left(M_{h}^{2}-M_{1}^{2}\right) \sqrt{\left(M_{h}^{2}-M_{1}^{2}\right)^{2}+4 M_{1 h}^{4}}\right] ; \\
& m_{1}^{2}=\frac{1}{2}\left[M_{h}^{2}+M_{1}^{2}-\varepsilon\left(M_{h}^{2}-M_{1}^{2}\right) \sqrt{\left(M_{h}^{2}-M_{1}^{2}\right)^{2}+4 M_{1 h}^{4}}\right],
\end{aligned}
$$

where $\varepsilon$ is the sign function.

Written now directly in terms of the physical fields, the cubic interactions are expressed as follows:

$$
U_{\text {cub }}=\frac{\lambda_{0}^{(3)}}{2} S_{0}^{2} h+\frac{\eta_{01}^{(3)}}{2} S_{0}^{2} S_{1}+\frac{\lambda^{(3)}}{6} h^{3}+\frac{\eta_{1}^{(3)}}{6} S_{1}^{3}+\frac{\lambda_{1}^{(3)}}{2} h^{2} S_{1}+\frac{\lambda_{2}^{(3)}}{2} h S_{1}^{2},
$$

where the cubic physical coupling constants are related to the original parameters via the following relations:

$$
\begin{aligned}
& \lambda_{0}^{(3)}=\lambda_{0} v \cos \theta+\eta_{01} v_{1} \sin \theta, \quad \eta_{01}^{(3)}=\eta_{01} v_{1} \cos \theta-\lambda_{0} v \sin \theta \\
& \lambda^{(3)}=\lambda v \cos ^{3} \theta+\frac{3}{2} \lambda_{1} \sin 2 \theta\left(v_{1} \cos \theta+v \sin \theta\right)+\eta_{1} v_{1} \sin ^{3} \theta \\
& \eta_{1}^{(3)}=\eta_{1} v_{1} \cos ^{3} \theta-\frac{3}{2} \lambda_{1} \sin 2 \theta\left(v \cos \theta-v_{1} \sin \theta\right)-\lambda v \sin ^{3} \theta \\
& \lambda_{1}^{(3)}=\lambda_{1} v_{1} \cos ^{3} \theta+\frac{1}{2} \sin 2 \theta\left[\left(2 \lambda_{1}-\lambda\right) v \cos \theta-\left(2 \lambda_{1}-\eta_{1}\right) v_{1} \sin \theta\right]-\lambda_{1} v \sin ^{3} \theta \\
& \lambda_{2}^{(3)}=\lambda_{1} v \cos ^{3} \theta-\frac{1}{2} \sin 2 \theta\left[\left(2 \lambda_{1}-\eta_{1}\right) v_{1} \cos \theta+\left(2 \lambda_{1}-\lambda\right) v \sin \theta\right]+\lambda_{1} v_{1} \sin ^{3} \theta
\end{aligned}
$$


Written too directly in terms of the physical fields, the quartic interactions are given by:

$$
\begin{aligned}
U_{\text {quar }}= & \frac{\eta_{0}}{24} S_{0}^{4}+\frac{\lambda^{(4)}}{24} h^{4}+\frac{\eta_{1}^{(4)}}{24} S_{1}^{4}+\frac{\lambda_{0}^{(4)}}{4} S_{0}^{2} h^{2}+\frac{\eta_{01}^{(4)}}{4} S_{0}^{2} S_{1}^{2}+\frac{\lambda_{01}^{(4)}}{2} S_{0}^{2} h S_{1} \\
& +\frac{\lambda_{1}^{(4)}}{6} h^{3} S_{1}+\frac{\lambda_{2}^{(4)}}{4} h^{2} S_{1}^{2}+\frac{\lambda_{3}^{(4)}}{6} h S_{1}^{3},
\end{aligned}
$$

where the physical quartic coupling constants are written in terms of the original parameters of the theory as follows:

$$
\begin{aligned}
& \lambda^{(4)}=\lambda \cos ^{4} \theta+\frac{3}{2} \lambda_{1} \sin ^{2} 2 \theta+\eta_{1} \sin ^{4} \theta, \quad \eta_{1}^{(4)}=\eta_{1} \cos ^{4} \theta+\frac{3}{2} \lambda_{1} \sin ^{2} 2 \theta+\lambda \sin ^{4} \theta \\
& \lambda_{0}^{(4)}=\lambda_{0} \cos ^{2} \theta+\eta_{01} \sin ^{2} \theta, \quad \eta_{01}^{(4)}=\eta_{01} \cos ^{2} \theta+\lambda_{0} \sin ^{2} \theta, \quad \lambda_{01}^{(4)}=\frac{1}{2}\left(\eta_{01}-\lambda_{0}\right) \sin 2 \theta \\
& \lambda_{1}^{(4)}=\frac{1}{2}\left[\left(3 \lambda_{1}-\lambda\right) \cos ^{2} \theta-\left(3 \lambda_{1}-\eta_{1}\right) \sin ^{2} \theta\right] \sin 2 \theta \\
& \lambda_{2}^{(4)}=\lambda_{1} \cos ^{2} 2 \theta-\frac{1}{4}\left(2 \lambda_{1}-\eta_{1}-\lambda\right) \sin ^{2} 2 \theta \\
& \lambda_{3}^{(4)}=\frac{1}{2}\left[\left(\eta_{1}-3 \lambda_{1}\right) \cos ^{2} \theta-\left(\lambda-3 \lambda_{1}\right) \sin ^{2} \theta\right] \sin 2 \theta
\end{aligned}
$$

In addition to the above sector and after spontaneous breaking of the electroweak and $\mathbb{Z}_{2}$ symmetries, we need to rewrite the part of the Standard Model lagrangian affected by the mixing angle $\theta$. We thus have:

$$
\begin{aligned}
U_{\mathrm{SM}}= & \sum_{f}\left(\lambda_{h f} h \bar{f} f+\lambda_{1 f} S_{1} \bar{f} f\right)+\lambda_{h w}^{(3)} h W_{\mu}^{-} W^{+\mu}+\lambda_{1 w}^{(3)} S_{1} W_{\mu}^{-} W^{+\mu} \\
& +\lambda_{h z}^{(3)} h\left(Z_{\mu}\right)^{2}+\lambda_{1 z}^{(3)} S_{1}\left(Z_{\mu}\right)^{2}+\lambda_{h w}^{(4)} h^{2} W_{\mu}^{-} W^{+\mu}+\lambda_{1 w}^{(4)} S_{1}^{2} W_{\mu}^{-} W^{+\mu} \\
& +\lambda_{h 1 w} h S_{1} W_{\mu}^{-} W^{+\mu}+\lambda_{h z}^{(4)} h^{2}\left(Z_{\mu}\right)^{2}+\lambda_{1 z}^{(4)} S_{1}^{2}\left(Z_{\mu}\right)^{2}+\lambda_{h 1 z} h S_{1}\left(Z_{\mu}\right)^{2} .
\end{aligned}
$$

The quantities $m_{f}, m_{w}$ and $m_{z}$ are the masses of the fermion $f$, the $W$ and the $Z$ gauge bosons respectively, and the above coupling constants are given by the following relations:

$$
\begin{array}{lll}
\lambda_{h f}=-\frac{m_{f}}{v} \cos \theta ; & \lambda_{1 f}=\frac{m_{f}}{v} \sin \theta ; \\
\lambda_{h w}^{(3)}=2 \frac{m_{w}^{2}}{v} \cos \theta ; & \lambda_{1 w}^{(3)}=-2 \frac{m_{w}^{2}}{v} \sin \theta ; \\
\lambda_{h z}^{(3)}=\frac{m_{z}^{2}}{v} \cos \theta ; & \lambda_{1 z}^{(3)}=-\frac{m_{z}^{2}}{v} \sin \theta ; \\
\lambda_{h w}^{(4)}=\frac{m_{w}^{2}}{v^{2}} \cos ^{2} \theta ; & \lambda_{1 w}^{(4)}=\frac{m_{w}^{2}}{v^{2}} \sin ^{2} \theta ; & \lambda_{h 1 w}=-\frac{m_{w}^{2}}{v^{2}} \sin 2 \theta ; \\
\lambda_{h z}^{(4)}=\frac{m_{z}^{2}}{2 v^{2}} \cos ^{2} \theta ; & \lambda_{1 z}^{(4)}=\frac{m_{z}^{2}}{2 v^{2}} \sin ^{2} \theta ; & \lambda_{h 1 z}=-\frac{m_{z}^{2}}{2 v^{2}} \sin 2 \theta .
\end{array}
$$




\section{Effects of the relic density constraint}

The original theory (2) has nine parameters: three mass parameters $\left(\tilde{m}_{0}, \mu, \mu_{1}\right)$, three self-coupling constants $\left(\eta_{0}, \lambda, \eta_{1}\right)$ and three mutual coupling constants $\left(\lambda_{0}, \eta_{01}, \lambda_{1}\right)$. The dark-matter self-coupling constant $\eta_{0}$ does not enter the calculations of the lowest-order processes to come?, so effectively, one is left with eight parameters. The spontaneous breaking of the electroweak and $\mathbb{Z}_{2}$ symmetries for the Higgs and $\chi_{1}$ fields respectively introduces the two vacuum expectation values $v$ and $v_{1}$ given to lowest order in (3). The value of $v$ is fixed experimentally to be $246 \mathrm{GeV}$ and we fix the value of $v_{1}$ at the order of the electroweak scale, say $100 \mathrm{GeV}$. So now six parameters left. It is natural to choose four of these the three physical masses $m_{0}$ (dark matter), $m_{1}$ ( $S_{1}$ field) and $m_{h}$ (Higgs), plus the mixing angle $\theta$ between $S_{1}$ and $h$. We give the Higgs mass the value $m_{h}=138 \mathrm{GeV}$, compatible with current experimental bounds. The two last parameters one chooses to work with are the two physical mutual coupling constants $\lambda_{0}^{(4)}$ (dark matter - Higgs) and $\eta_{01}^{(4)}$ (dark matter - $S_{1}$ particle), see (13). The thermal dynamics of the Universe within the standard cosmological model Kolb \& Turner (1998) relates the WIMP relic density $\Omega_{\mathrm{DM}}$ to its annihilation rate by two relations, which are essentially model independent:

$$
\Omega_{\mathrm{DM}} \bar{h}^{2} \simeq \frac{1.07 \times 10^{9} x_{f}}{\sqrt{g_{*}} m_{\mathrm{Pl}}\left\langle v_{12} \sigma_{\mathrm{ann}}\right\rangle \mathrm{GeV}} ; \quad x_{f} \simeq \ln \frac{0.038 m_{\mathrm{Pl}} m_{0}\left\langle v_{12} \sigma_{\mathrm{ann}}\right\rangle}{\sqrt{\bar{\delta}_{*} x_{f}}} .
$$

The notation is as follows: the quantity $\bar{h}$ is the Hubble constant in units of $100 \mathrm{~km} \times \mathrm{s}^{-1} \times$ $\mathrm{Mpc}^{-1}$, the quantity $m_{\mathrm{Pl}}=1.22 \times 10^{19} \mathrm{GeV}$ the Planck mass, $m_{0}$ the WIMP (dark matter) mass, $x_{f}=m_{0} / T_{f}$ the ratio of the WIMP mass to the freeze-out temperature $T_{f}$ and $g_{*}$ the number of relativistic degrees of freedom with mass less than $T_{f}$. The quantity $\left\langle v_{12} \sigma_{\text {ann }}\right\rangle$ is the thermally averaged annihilation cross-section of a pair of two dark-matter particles multiplied by their relative speed in the center-of-mass reference frame. Solving (17) with the current accepted value (1) for $\Omega_{\mathrm{DM}}$ yields a constraint on the annihilation cross-section, i.e.:

$$
\left\langle v_{12} \sigma_{\mathrm{ann}}\right\rangle \simeq(1.9 \pm 0.2) \times 10^{-9} \mathrm{GeV}^{-2} .
$$

In a given model like the one presented here, the above constraint translates into a relation between the parameters of the theory entering the calculated expression of $\left\langle v_{12} \sigma_{\text {ann }}\right\rangle$, hence limiting the intervals of possible dark matter masses. This constraint can also be exploited in order to examine aspects of the theory like perturbativity, while at the same time reducing the number of parameters by one. For example, in this model, we can use (18) to obtain the mutual coupling constant $\eta_{01}^{(4)}$ as a function of the remaining four parameters $\left(m_{0}, m_{1}, \theta, \lambda_{0}^{(4)}\right)$ and study aspects of the model through its behavior. For example, we can ask which dark-matter mass regions are consistent with perturbativity. Note that through the relations (12) and (14), once the two mutual coupling constants $\lambda_{0}^{(4)}$ and $\eta_{01}^{(4)}$ are perturbative, all the other physical coupling constants will be.

The dark-matter annihilation cross sections (times the relative speed) through all possible channels within the model can be calculated in the usual manner to lowest order in perturbation theory Abada (2011). The quantity $\left\langle v_{12} \sigma_{\text {ann }}\right\rangle$ is the sum of all these contributions. Imposing $\left\langle v_{12} \sigma_{\text {ann }}\right\rangle=1.9 \times 10^{-9} \mathrm{GeV}^{-2}$ dictates the behavior of $\eta_{01}^{(4)}$, which is displayed as a function of the dark matter mass $m_{0}$. Of course, as there are four free parameters, the behavior is bound to be rich and diverse and we cannot describe every bit of it in such a small space. 
Also, importantly enough, one has to note from the outset that for a given set of values of the parameters, the solution to the relic-density constraint is not unique: besides positive real solutions (when they exist), we may find negative real or even complex solutions. Indeed, from the physical coefficients in (12) and (14), one can show that $\left\langle v_{12} \sigma_{\text {ann }}\right\rangle$ is a sum of quotients of up-to-quartic polynomials in $\eta_{01}^{(4)}$. This means that, ultimately, the relic-density constraint is going to be an algebraic equation in $\eta_{01}^{(4)}$, which has always solutions in the complex plane, but not necessarily on the positive real axis. In our context, we are only interested in finding the smallest of the positive real solutions in $\eta_{01}^{(4)}$ when they exist, looking at its behavior and finding out in which mass regions it is small enough to be perturbative.

We start the description with a small mixing angle, say $\theta=10^{\circ}$, and a very weak mutual $S_{0}$ - Higgs coupling constant, say $\lambda_{0}^{(4)}=0.01$. The behavior of $\eta_{01}^{(4)}$ versus $m_{0}$ for the $S_{1}$ mass $m_{1}=10 \mathrm{GeV}$ is displayed in Fig. 1 . The range of $m_{0}$ shown is wide, from $0.1 \mathrm{GeV}$ to

$$
\theta=10^{\circ}, \lambda_{0}{ }^{(4)}=0.01, m_{1}=10 \mathrm{GeV}
$$

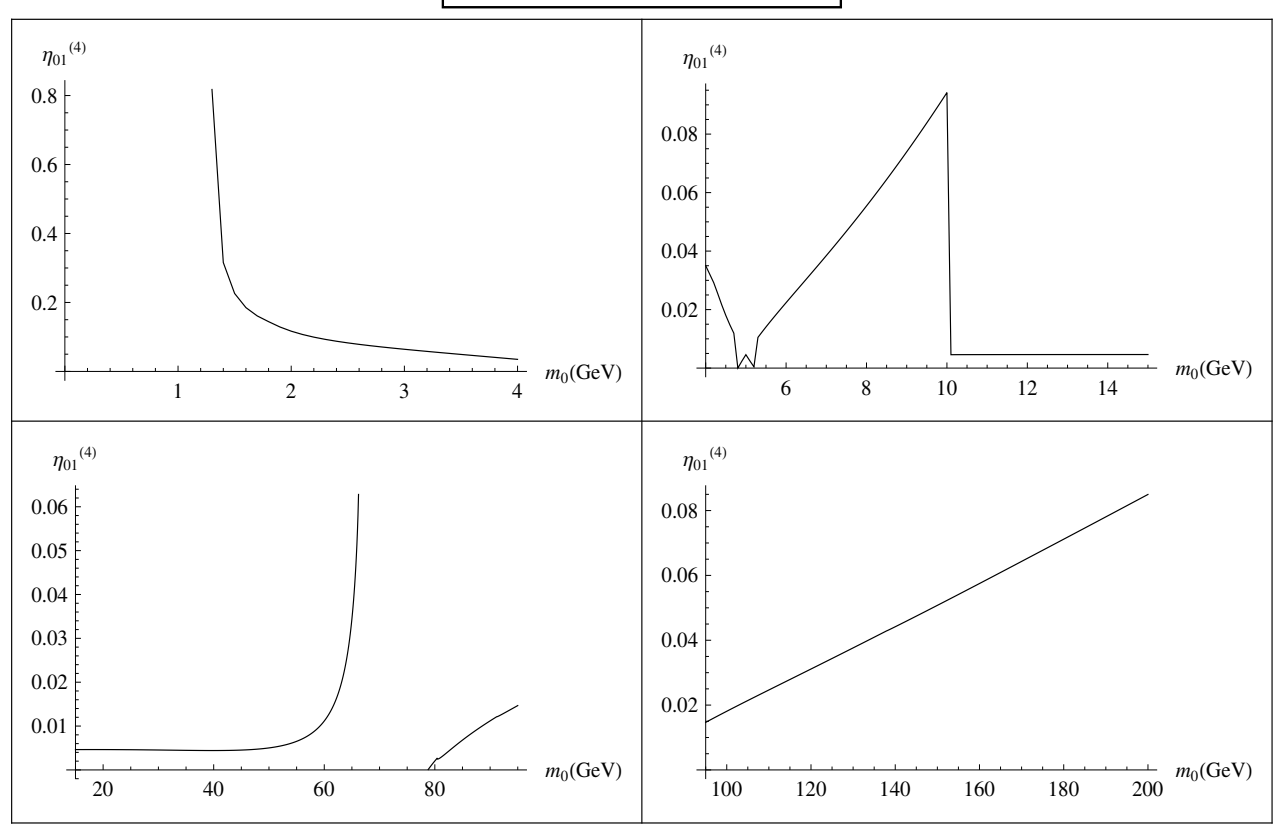

Fig. 1. $\eta_{01}^{(4)}$ vs $m_{0}$ for small $m_{1}$, small mixing and very small WIMP-Higgs coupling.

$200 \mathrm{GeV}$, cut in four intervals to allow for 'local' features to be displayed. We see that the relic-density constraint on $S_{0}$ annihilation has no positive real solution for $m_{0} \lesssim 1.3 \mathrm{GeV}$, and so, with these very small masses, $S_{0}$ cannot be a dark matter candidate. In other words, for $m_{1}=10 \mathrm{GeV}$, the particle $S_{0}$ cannot annihilate into the lightest fermions only in a way compatible with the relic-density constraint; inclusion of the $c$-quark is necessary. Note that right about $m_{0} \simeq 1.3 \mathrm{GeV}$, the $c$ threshold, the mutual coupling constant $\eta_{01}^{(4)}$ starts at about 0.8 , a value, while perturbative, that is roughly eighty-fold larger than the mutual $S_{0}$ - Higgs coupling constant $\lambda_{0}^{(4)}$. Then $\eta_{01}^{(4)}$ decreases, steeply first, more slowly as we cross the $\tau$ mass 
towards the $b$ mass. Just before $m_{1} / 2$, the coupling $\eta_{01}^{(4)}$ hops onto another solution branch that is just emerging from negative territory, gets back to the first one at precisely $m_{1} / 2$ as this latter carries now smaller values, and then jumps up again onto the second branch as the first crosses the $m_{0}$-axis down. It goes up this branch with a moderate slope until $m_{0}$ becomes equal to $m_{1}$, a value at which the $S_{1}$ annihilation channel opens. Right beyond $m_{1}$, there is a sudden fall to a value $\eta_{01}^{(4)} \simeq 0.0046$ that is about half the value of $\lambda_{0}^{(4)}$, and $\eta_{01}^{(4)}$ stays flat till $m_{0} \simeq 45 \mathrm{GeV}$ where it starts increasing, sharply after $60 \mathrm{GeV}$. In the mass interval $m_{0} \simeq 66 \mathrm{GeV}-79 \mathrm{GeV}$, there is a 'desert' with no positive real solutions to the relic-density constraint, hence no viable dark matter candidate. Beyond $m_{0} \simeq 79 \mathrm{GeV}$, the mutual coupling constant $\eta_{01}^{(4)}$ keeps increasing monotonously, with a small notch at the $W$ mass and a less noticeable one at the $Z$ mass.

For this value of $m_{1}(10 \mathrm{GeV})$, all values reached by $\eta_{01}^{(4)}$ in the mass range considered are perturbativily acceptable. This may not be the case for larger values of $m_{1}$. For example, for $m_{1}=30 \mathrm{GeV}$ while keeping $\theta=10^{\circ}$ and $\lambda_{0}^{(4)}=0.01$, the mutual coupling constant $\eta_{01}^{(4)}$ starts at $m_{0} \simeq 1.5 \mathrm{GeV}$ with the very large value 89.8 and decreases very sharply right after, to 2.04 at about $1.6 \mathrm{GeV}$. The other overall features are similar to the case $m_{1}=10 \mathrm{GeV}$.

One important question to ask is whether the model ever allows for very light dark matter. To look into this matter, one fixes $m_{0}$ at a small value, say $m_{0}=0.2 \mathrm{GeV}$, and let $m_{1}$ vary. The behavior of $\eta_{01}^{(4)}$ is displayed in Fig. 2. The allowed $S_{0}$ annihilation channels are the very light fermions $e, u, d, \mu$ and $s$, plus $S_{1}$ when $m_{1}<m_{0}$. Qualitatively, we notice that in fact, there are no solutions for $m_{1}<m_{0}$, a mass at which $\eta_{01}^{(4)}$ takes the very small value $\simeq 0.003$. It goes up a solution branch and leaves it at $m_{1} \simeq 0.4 \mathrm{GeV}$ to descend on a second branch that enters negative territory at $m_{1} \simeq 0.7 \mathrm{GeV}$, forcing $\eta_{01}^{(4)}$ to return onto the first branch. There is an accelerated increase till $m_{1} \simeq 5 \mathrm{GeV}$, a value at which $\eta_{01}^{(4)} \simeq 0.5$. And then a desert, no positive real solutions, no viable dark matter.

$$
\theta=10^{\circ}, \lambda_{0}^{(4)}=0.01, m_{0}=0.2 \mathrm{GeV}
$$

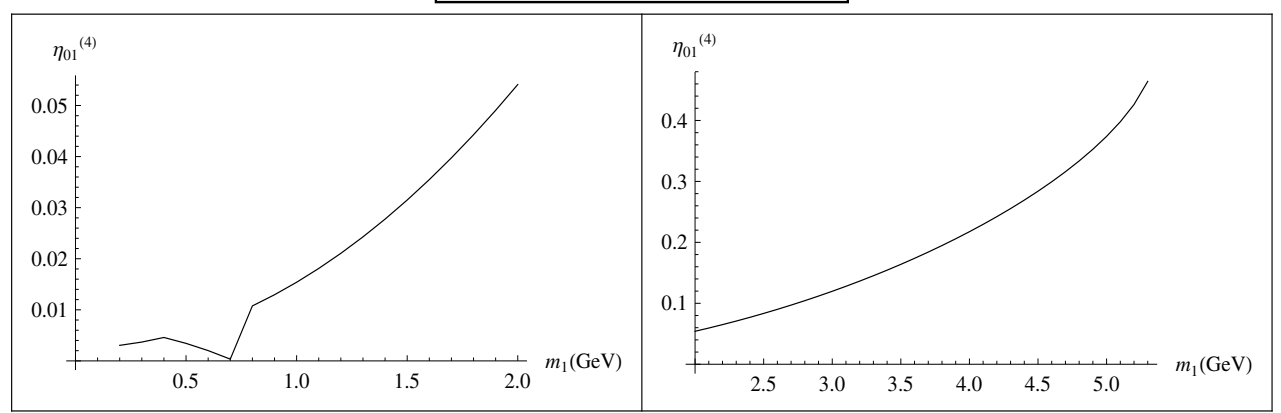

Fig. 2. $\eta_{01}^{(4)}$ vs $m_{1}$ for very light $S_{1}$, small mixing and very small WIMP-Higgs coupling.

Increasing $m_{0}$ until about $1.3 \mathrm{GeV}$ does not change these overall features: some 'movement' for very small values of $m_{1}$ and then an accelerated increase till reaching a desert with a lower bound that changes with $m_{0}$. Note that in all these cases where $m_{0} \lesssim 1.3 \mathrm{GeV}$, all values of $\eta_{01}^{(4)}$ are perturbative. Therefore, the model can very well accommodate very light dark matter with a restricted range of $S_{1}$ masses. However, the situation changes after the inclusion of 
the $\tau$ annihilation channel. Indeed, though the overall shape of the behavior of $\eta_{01}^{(4)}$ as a function of $m_{1}$ is qualitatively the same, the desert threshold is pushed significantly higher, and more significantly, $\eta_{01}^{(4)}$ starts to be larger than one already at moderately small values of $m_{1}$, therefore loosing perturbativity. In fact, for $m_{0}=1.5 \mathrm{GeV}$ already, the desert is effectively erased as we have a sudden jump to highly non-perturbative values of $\eta_{01}^{(4)}$ right after $m_{1} \simeq$ $28 \mathrm{GeV}$ ?. However, for $m_{1}$ moderately small, for example $\lesssim 20 \mathrm{GeV}$ in the case $m_{0}=1.5 \mathrm{GeV}$, the values of $\eta_{01}^{(4)}$ are smaller than one and physical use of the model is possible if needed.

Some new features come when increasing the value of the mutual coupling constant $\lambda_{0}^{(4)}$. Figure 3 shows the behavior of $\eta_{01}^{(4)}$ as a function of the dark matter mass $m_{0}$ when $\lambda_{0}^{(4)}=0.2$, $\theta=10^{\circ}$ and $m_{1}=20 \mathrm{GeV}$. We see that $\eta_{01}^{(4)}$ starts at $m_{0} \simeq 1.4 \mathrm{GeV}$ with a value of about 1.95 . It decreases with a sharp change of slope at the $b$ threshold, then makes a sudden dive at about $5 \mathrm{GeV}$, a change of branch at $m_{1} / 2$ down till about $12 \mathrm{GeV}$ where it jumps up back onto the previous branch just before going to cross into negative territory. It drops sharply at $m_{0}=m_{1}$ and then increases slowly until $m_{0} \simeq 43.3 \mathrm{GeV}$. Beyond, there is nothing, a desert. This is of course different from the situation of very small $\lambda_{0}^{(4)}$ like in Fig. 1 above: here we see some kind of natural dark-matter mass 'confinement' to small-moderate viable ${ }^{2}$ values.

$$
\theta=10^{\circ}, \lambda_{0}{ }^{(4)}=0.2, m_{1}=20 \mathrm{GeV}
$$

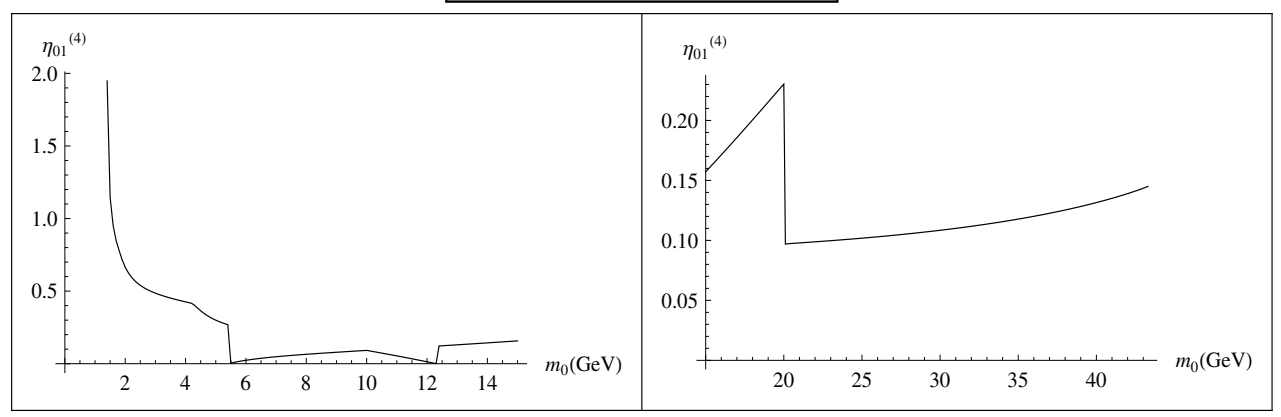

Fig. 3. $\eta_{01}^{(4)}$ vs $m_{0}$ for small mixing, moderate $m_{1}$ and WIMP-Higgs coupling.

For larger values of $m_{1}$ with moderate $\lambda_{0}^{(4)}=0.2$, one obtains roughly the same behavior but here too not all values of $\eta_{01}^{(4)}$ are perturbative. For example, for $m_{1}=60 \mathrm{GeV}$, the mutual coupling $\eta_{01}^{(4)}$ starts very high $(\simeq 85)$ at $m_{0} \simeq 1.5 \mathrm{GeV}$, and then decreases rapidly. There is a usual change of branches and a desert starting at about $49 \mathrm{GeV}$. However, what is interesting here is that, in contrast with previous situations, the desert starts at a mass $m_{0}<m_{1}$, i.e., before the opening of the $S_{1}$ annihilation channel. In other words, the dark matter is annihilating into the light fermions only and the model is perturbatively viable in the range $20 \mathrm{GeV}-49 \mathrm{GeV}$.

Larger values of $\lambda_{0}^{(4)}$ can also be studied. For $\lambda_{0}^{(4)}=1$ and as long as $m_{1} \lesssim 79.2 \mathrm{GeV}$, one finds the usual small $m_{0}$-deserts as well as the familar action at the different mass thresholds, with nothing suprisingly new. However, for $m_{1} \gtrsim 79.3 \mathrm{GeV}$, there is a highly non-perturbative

\footnotetext{
${ }^{2}$ Note that the values of $\eta_{01}^{(4)}$ for $1.6 \mathrm{GeV} \lesssim m_{0} \lesssim 43.3 \mathrm{GeV}$ are all perturbative.
} 
branch $\eta_{01}^{(4)}$ jumps onto at small and moderate values of $m_{0}$ ?. This highly non-perturbative region stretches in size as $m_{1}$ increases.

Increasing the $S_{1}$ - Higgs mixing angle $\theta$ can bring new features too. Figure 4 shows the behavior of $\eta_{01}^{(4)}$ as a function of $m_{0}$ for $\theta=40^{\circ}, \lambda_{0}^{(4)}=0.01$ and $m_{1}=20 \mathrm{GeV}$. One recognizes features similar to those of the case $\theta=10^{\circ}$, though coming in different relative sizes. The very-small- $m_{0}$ desert ends at about $0.3 \mathrm{GeV}$. There are by-now familiar features at the $c$ and $b$ masses, $m_{1} / 2$ and $m_{1}$. Two relatively small forbidden intervals (deserts) appear for relatively large values of the dark matter mass: $67.3 \mathrm{GeV}-70.9 \mathrm{GeV}$ and $79.4 \mathrm{GeV}-90.8 \mathrm{GeV}$. The $W$ mass is in the forbidden region but there is action as we cross the $Z$ mass. Other values of $m_{1}$ behave similarly with an additional effect, namely, a sudden drop in slope at $m_{0}=$ $\left(m_{h}+m_{1}\right) / 2$ coming from the ignition of $S_{0}$ annihilation into $S_{1}$ and Higgs.

$$
\theta=40^{\circ}, \lambda_{0}{ }^{(4)}=0.01, m_{1}=20 \mathrm{GeV}
$$

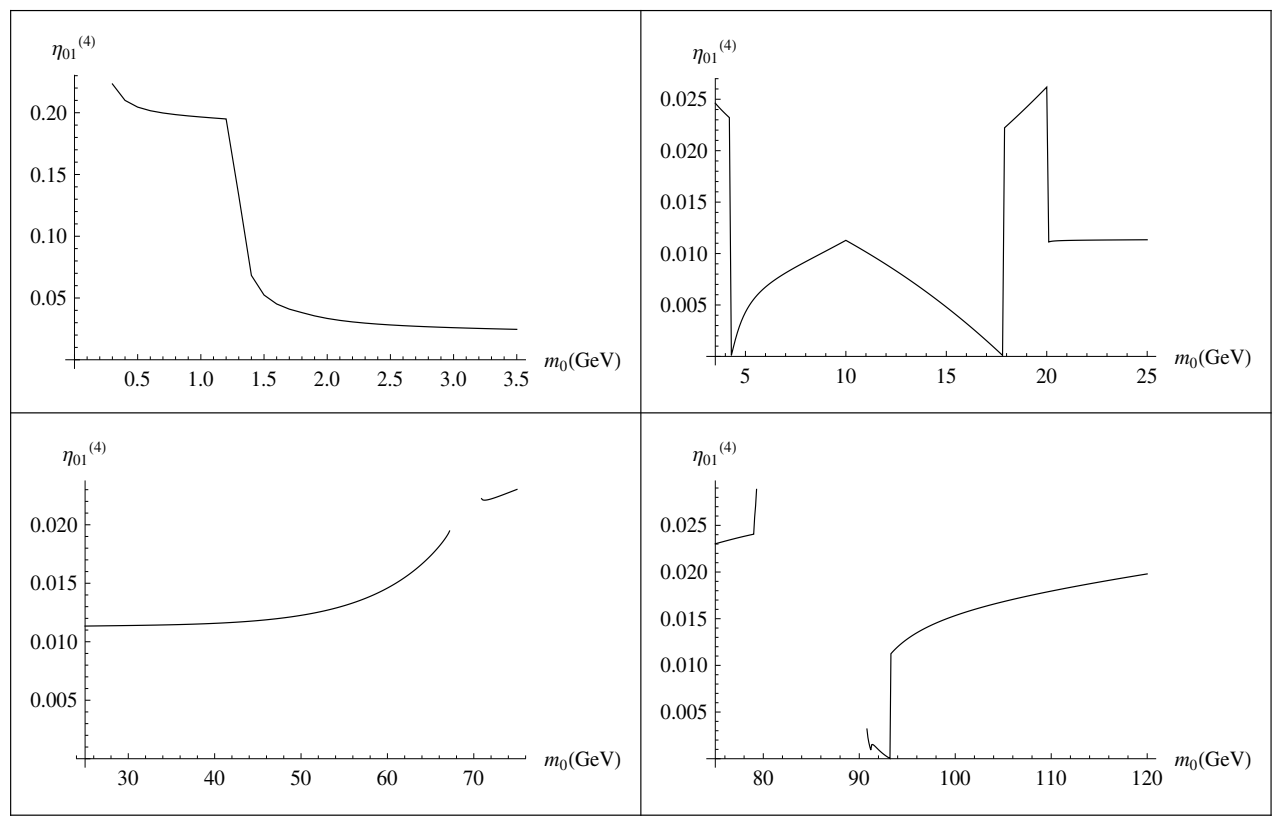

Fig. 4. $\eta_{01}^{(4)}$ versus $m_{0}$ for moderate $m_{1}$, moderate mixing and small WIMP-Higgs coupling.

Increasing the value of $\lambda_{0}^{(4)}$ for larger values of $\theta$ has the effect of making the behavior of $\eta_{01}^{(4)}$ smoother while keeping the same overall features like the confining of the mass of a viable dark matter to small-moderate values, a dark matter particle annihilating into light fermions only. It has also the effect of eliminating those highly non-perturbative regions discussed above.

\section{Dark-matter direct detection}

Experiments like CDMS II Ahmed et al. (2009), XENON 10/100 Angle et al. (2008); ?, DAMA/LIBRA Bernabei et al. (2010) and CoGeNT Aalseth et al. (2010) carry a direct search 
for a dark matter signal. Such a signal would typically come from the elastic scattering of a dark matter WIMP off a non-relativistic nucleon target. However, such experiments have not yet detected an unambiguous signal, but rather yielded increasingly stringent exclusion bounds on the dark matter - nucleon elastic-scattering total cross-section $\sigma_{\text {det }}$ in terms of the dark matter mass $m_{0}$.

Therefore, in order to see if the present two-singlet extension of the Standard Model is a viable dark matter model, we have to calculate $\sigma_{\text {det }}$ as a function of $m_{0}$ for different values of the parameters $\left(\theta, \lambda_{0}^{(4)}, m_{1}\right)$ and project its behavior against the experimental bounds. We will limit ourselves to the region $0.1 \mathrm{GeV}-100 \mathrm{GeV}$ as we are interested in light dark matter only. As experimental bounds, we will use the results from CDMSII and XENON100, as well as the future projections of SuperCDMS Schnee et al. (2005) and XENON1T April et al. (2010). As the figures below show (Gaitskell et al., 2011), in the region of our interest, XENON100 is only slightly tighter than CDMSII, SuperCDMS significantly lower and XENON1T the most stringent by far. But it is important to note that all these results loose reasonable predictability in the very light sector, say below $5 \mathrm{GeV}$.

The scattering of $S_{0}$ off a SM fermion $f$ occurs via the t-channel exchange of the SM Higgs and $S_{1}$. In the non-relativistic limit, the effective Lagrangian describing this scattering reads:

$$
\mathcal{L}_{S_{0}-f}^{(\text {eff })}=a_{f} S_{0}^{2} \bar{f} f,
$$

where the coupling constant $a_{f}$ is related to the physical parameters of the theory by the following relation:

$$
a_{f}=-\frac{m_{f}}{2 v}\left[\frac{\lambda_{0}^{(3)} \cos \theta}{m_{h}^{2}}-\frac{\eta_{01}^{(3)} \sin \theta}{m_{1}^{2}}\right] .
$$

From this interaction, we calculate the total cross-section for this scattering process and find:

$$
\sigma_{S_{0} f \rightarrow S_{0} f}=\frac{m_{f}^{4}}{4 \pi\left(m_{f}+m_{0}\right)^{2} v^{2}}\left[\frac{\lambda_{0}^{(3)} \cos \theta}{m_{h}^{2}}-\frac{\eta_{01}^{(3)} \sin \theta}{m_{1}^{2}}\right]^{2} .
$$

At the nucleon level, the effective interaction Lagrangian between $S_{0}$ and a nucleon $N=p$ or $n$ has the form:

$$
\mathcal{L}_{S_{0}-N}^{(\text {eff })}=a_{N} S_{0}^{2} \bar{N} N
$$

where the effective $S_{0}$ - nucleon coupling constant $a_{N}$ is given by the relation:

$$
a_{N}=\frac{\left(m_{N}-\frac{7}{9} m_{B}\right)}{v}\left[\frac{\lambda_{0}^{(3)} \cos \theta}{m_{h}^{2}}-\frac{\eta_{01}^{(3)} \sin \theta}{m_{1}^{2}}\right]
$$

where $m_{N}$ is the nucleon mass and $m_{B}$ the baryon mass in the chiral limit ?. The total cross section for non-relativistic $S_{0}-N$ elastic scattering is therefore:

$$
\sigma_{\text {det }} \equiv \sigma_{S_{0} N \rightarrow S_{0} N}=\frac{m_{N}^{2}\left(m_{N}-\frac{7}{9} m_{B}\right)^{2}}{4 \pi\left(m_{N}+m_{0}\right)^{2} v^{2}}\left[\frac{\lambda_{0}^{(3)} \cos \theta}{m_{h}^{2}}-\frac{\eta_{01}^{(3)} \sin \theta}{m_{1}^{2}}\right]^{2} .
$$


Let us briefly discuss the behavior of $\sigma_{\text {det }}$ as a function of $m_{0}$ for an indicative set of values of the parameters $\left(\theta, \lambda_{0}^{(4)}, m_{1}\right)$. Of course, we have to impose systematically the relic-density constraint on the dark matter annihilation cross-section (18). But in addition, we will require here that the coupling constants are perturbative, and so impose the additional requirement $0 \leq \eta_{01}^{(4)} \leq 1$. Also, before getting into some details, let us quickly mention some global trends in the behavior of the detection cross-section. Generally, as $m_{0}$ increases, the detection cross-section $\sigma_{\mathrm{det}}$ starts from high values, slopes down to minima that depend on the parameters and then picks up moderately. There are features and action at the usual mass thresholds, with varying sizes and shapes. Excluded regions are there, those coming from the relic-density constraint and new ones originating from the additional perturbativity requirement. Close to the upper boundary of the mass interval considered in this study, there is no universal behavior to mention as in some cases $\sigma_{\text {det }}$ will increase monotonously and, in some others, it will decrease or 'not be there' at all.

For a small Higgs $-S_{1}$ mixing angle, say $\theta=10^{\circ}$, and a very weak mutual $S_{0}$ - Higgs coupling, $\lambda_{0}^{(4)}=0.01$, the behavior of $\sigma_{\text {det }}$ is displayed in figure 5 where $m_{1}=20 \mathrm{GeV}$. We see that for the two mass intervals $20 \mathrm{GeV}-65 \mathrm{GeV}$ and $75 \mathrm{GeV}-100 \mathrm{GeV}$, plus an almost singled-out dip at $m_{0}=m_{1} / 2$, the elastic scattering cross section is below the projected sensitivity of SuperCDMS. However, XENON1T will probe all these masses except for $m_{0} \simeq 58 \mathrm{GeV}$ and $85 \mathrm{GeV}$.

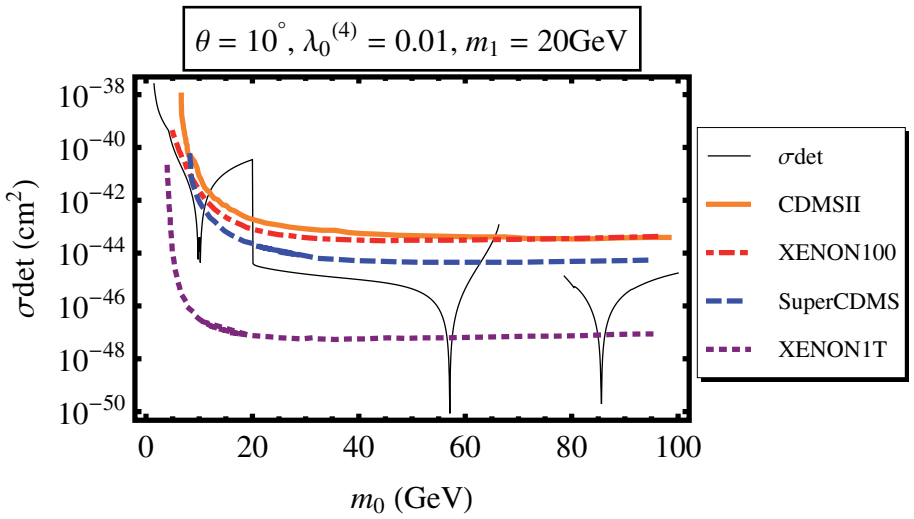

Fig. 5. Elastic $N-S_{0}$ scattering cross-section as a function of $m_{0}$ for moderate $m_{1}$, small mixing and small WIMP-Higgs coupling.

Also, as we see in Fig. 5, most of the mass range for very light dark matter is excluded for these values of the parameters. Is this systematic? In general, smaller values of $m_{1}$ drive the predictability ranges to the lighter sector of the dark matter masses. Figure 6 illustrates this pattern. We have taken $m_{1}=5 \mathrm{GeV}$, just above the lighter-quarks threshold. In the small-mass region, we see that SuperCDMS is passed in the range $5 \mathrm{GeV}-30 \mathrm{GeV}$. Here too, all this mass range will be probed by the XENON1T experiment, except a sharp dip at $m_{0}=m_{1} / 2=$ $2.5 \mathrm{GeV}$, but for such a very light mass, the experimental results are not without ambiguity. Reversely, increasing $m_{1}$ shuts down possibilities for very light dark matter and thins the intervals as it drives the predicted masses to larger values Abada (2011). 


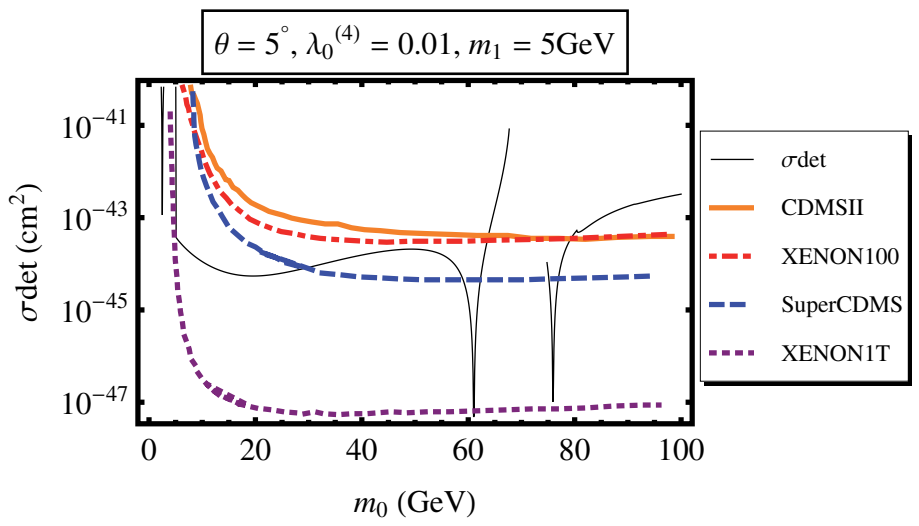

Fig. 6. Elastic $N-S_{0}$ scattering cross-section as a function of $m_{0}$ for light $S_{1}$, small mixing and small WIMP-Higgs coupling.

A larger mutual coupling constant $\lambda_{0}^{(4)}$ has the general effect of squeezing the acceptable intervals of $m_{0}$ by pushing the values of $\sigma_{\text {det }}$ up. Also, increasing the mixing angle $\theta$ has the general effect of increasing the value of $\sigma_{\mathrm{det}}$. Figure 7 shows this trend for $\theta=40^{\circ}$; compare with Fig. 5. The only allowed masses by the current bounds of CDMSII and XENON100 are between $20 \mathrm{GeV}$ and $50 \mathrm{GeV}$, the narrow interval around $m_{1} / 2$, and another very sharp one, at about $94 \mathrm{GeV}$. The projected sensitivity of XENON1T will probe all these mases except those at $m_{0} \simeq 30 \mathrm{GeV}$ and $94 \mathrm{GeV}$. Finally, there are regions of the parameters for which the model has no predictability. This can happen when we combine the effects of increasing the values of the two parameters $\lambda_{0}^{(4)}$ and $m_{1}$.

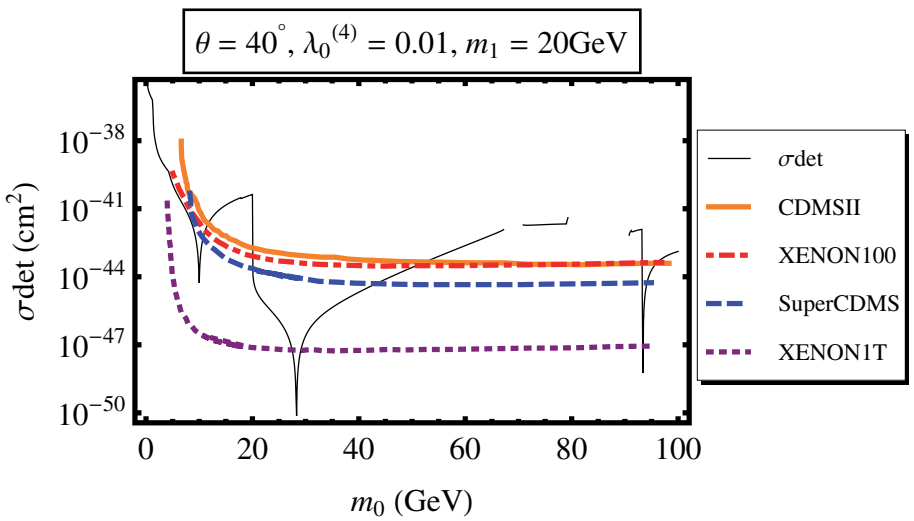

Fig. 7. Elastic $N-S_{0}$ scattering cross-section as a function of $m_{0}$ for moderate $m_{1}$, large mixing and small WIMP-Higgs coupling. 


\section{Constraints from phenomenology}

Besides its direct scattering off a nucleon, a light dark-matter WIMP can manifest itself in various low-energy processes. Possible delectability puts restrictions on the various parameters of a model like the one presented here. In this section, we illustrate this mechanism with an example ? and limit ourselves to low dark-matter masses, say from $0.1 \mathrm{GeV}-10 \mathrm{GeV}$. To ensure applicability of perturbation theory, the requirement $\eta_{01}^{(4)}<1$ is here too imposed throughout, together with a choice of weak values for $\lambda_{0}^{(4)}$. Finally, all particle data used in the sequel is taken from (Nakamura et al., 2010).

The process we consider is the decay of the $\bar{b} s$ bound state $B_{S}$ into, predominately, a pair of $\mu^{+} \mu^{-}$. The two corresponding SM diagrams sum up to yield a branching ratio $\mathrm{B}^{(\mathrm{SM})}\left(B_{S} \rightarrow \mu^{+} \mu^{-}\right)=(3.4 \pm 0.5) \times 10^{-9}$, whereas the experimental value is $\mathrm{B}^{(\exp )}\left(B_{S} \rightarrow \mu^{+} \mu^{-}\right) \simeq 4.7 \times 10^{-8}$. It means there is room for non-SM (invisible) processes to consider. In this two-singlet extension of the Standard Model, two additional decay diagrams occur, both via $S_{1}$ exchange, yielding together the branching ratio:

$$
\mathrm{B}^{\left(S_{1}\right)}\left(B_{s} \rightarrow \mu^{+} \mu^{-}\right)=\frac{9 \tau_{B_{s}} G_{F}^{4} f_{B_{s}}^{2} m_{B_{s}}^{5}}{2048 \pi^{5}} m_{\mu}^{2} m_{t}^{4}\left|V_{t b} V_{t s}^{*}\right|^{2} \frac{\left(1-4 m_{\mu}^{2} / m_{B_{s}}^{2}\right)^{3 / 2}}{\left(m_{B_{s}}^{2}-m_{1}^{2}\right)^{2}+m_{1}^{2} \Gamma_{1}^{2}} \sin ^{4} \theta .
$$

The particle data appearing in this expression are the $B_{s}$ life-time $\tau_{B_{s}}=1.43 \mathrm{ps}$, its mass $m_{B_{s}}=$ $5.366 \mathrm{GeV}$, the Fermi coupling constant $G_{F}$, the $B_{s}$ form factor $f_{B_{s}}$ that we take to be $210 \mathrm{MeV}$, the muon ( $t$-quark) mass $m_{\mu(t)}$, and the CKM elements $V_{t b}$ and $V_{t s}$. The quantity $\Gamma_{1}$ is the decay rate of the particle $S_{1}$.

This process depends directly on $m_{1}$ and the mixing angle $\theta$, whereas $m_{0}$ and the mutual coupling $\lambda_{0}^{(4)}$ enter (25) via the decay rate $\Gamma_{1}$. A generic behavior of $\mathrm{B}^{\left(S_{1}\right)}\left(B_{s} \rightarrow \mu^{+} \mu^{-}\right)$is shown in Fig. 8. Figure (L) shows the region (in gray) in the $\left(m_{1}, \theta\right)$ plane for which $\mathbf{B}^{\left(S_{1}\right)}$ is below the experimental value. The white narrow band about $m_{B_{s}}$ is what is excluded by $\mathrm{B}^{(\exp )}$, whereas the white zone on the left is lost to the relic-density constraint and perturbativity requirement. Varying $m_{0}$ in the range $0.1 \mathrm{GeV}-10 \mathrm{GeV}$ and $\lambda_{0}^{(4)}$ in the interval $0.01-0.9$ has little direct effect on the behavior of $\mathrm{B}^{\left(S_{1}\right)}$ as a function of $m_{1}$ and $\theta$, but does affect the relic-density constraint and perturbativity exclusion zones in their shapes, sizes and positions. Aside from these exclusion zones, most of the rest of the area is generically within the experimental bound, which means, in this sense, this process is not very restrictive by itself.

Figure ( $R$ ) on the right in Fig. 8 shows the regions (in gray) for which $\mathrm{B}^{\left(S_{1}\right)}$ is squeezed between $\mathrm{B}^{(\exp )}$ from above and the Standard Model prediction $\mathrm{B}^{(\mathrm{SM})}+3 \sigma$ from below, thus targetting an unambiguous signal if any. The behavior we see in this figure is generic across the ranges of $m_{0}$ and $\lambda_{0}^{(4)}$ : the V-shape structure in gray developing from $m_{1}=m_{B_{s}}$ is the allowed region. The white region in the middle is due the $\mathrm{B}^{(\exp )}$ and the white region outside is due to $\mathrm{B}^{(\mathrm{SM})}+3 \sigma$. It can happen that some of the gray $\mathrm{V}$ is eaten up by the relic-density constraint and perturbativity requirement for larger values of $\lambda_{0}^{(4)}$.

Once a region is gray on figure $(\mathrm{R})$, one has to check whether the dark-matter direct detection is allowed for the corresponding parameters. Remember that the constraint from relic density is applied systematically. Bearing in mind that the existing and predicted experimental bounds have no predictability for masses $0.1 \mathrm{GeV} \leq m_{0} \leq 5 \mathrm{GeV}$, we have checked that the direct 
detection cross-section is between SuperCDMS and Xenon1T for all gray points in figure (R), and this stays true for most values of $m_{0}$ and small $\lambda_{0}^{(4)}$. Therefore, there is no significant additional exclusion from direct detection.

From this process, there is probably one element to retain if we want the model to contribute a distinct signal to $B_{S} \rightarrow \mu^{+} \mu^{-}$for the range of $m_{0}$ chosen, and that it to restrict $4.8 \mathrm{GeV} \lesssim m_{1} \lesssim$ $6.2 \mathrm{GeV}$ and $\theta \gtrsim 8^{\circ}$. No additional constraint on $m_{0}$ is necessary while keeping $\lambda_{0}^{(4)} \lesssim 0.1$ to avoid systematic exclusion from direct detection is safe.
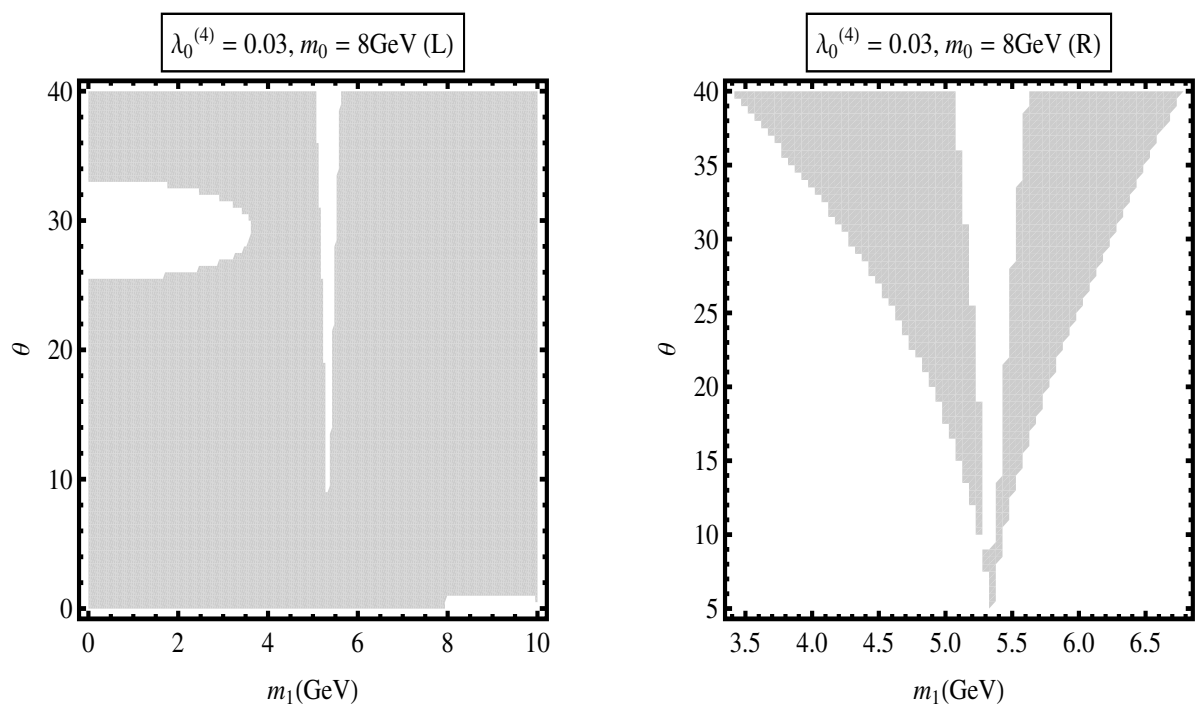

Fig. 8. The branching ratio (in gray) $\mathrm{B}^{\left(S_{1}\right)}\left(B_{S} \rightarrow \mu^{+} \mu^{-}\right) \leq \mathrm{B}^{(\exp )}$ in the left figure (L), and $\mathrm{B}^{(\mathrm{SM})}+3 \sigma \leq \mathrm{B}^{\left(S_{1}\right)}\left(B_{S} \rightarrow \mu^{+} \mu^{-}\right) \leq \mathrm{B}^{(\exp )}$ in (R). The angle $\theta$ is in degrees.

\section{Concluding remarks}

In this chapter, we have tried to show how a plausible scenario can model light cold dark matter. The model consists in enlarging the Standard Model with two gauge-singlet $\mathbb{Z}_{2}$-symmetric scalar fields. One is the dark matter field $S_{0}$, stable, while the other undergoes spontaneous symmetry breaking, resulting in the physical field $S_{1}$. The goal is to open additional channels through which $S_{0}$ can annihilate, hence reducing its number density. The model is parametrized by three quantities: the physical mutual coupling constant $\lambda_{0}^{(4)}$ between $S_{0}$ and the Higgs, the mixing angle $\theta$ between $S_{1}$ and the Higgs and the mass $m_{1}$ of the particle $S_{1}$.

We have carried our analysis in three steps. First we have imposed on the annihilation cross-section of $S_{0}$ the constraint from the observed dark-matter relic density and looked at its effects through the behavior of the physical mutual coupling constant $\eta_{01}^{(4)}$ between $S_{0}$ and $S_{1}$ as a function of the dark matter mass $m_{0}$. Apart from forbidden regions (deserts) and others where perturbativity is lost, we find that for most values of the three parameters, there 
are viable solutions in the small-moderate mass ranges of the dark matter sector. Deserts are found for most of the ranges of the parameters whereas perturbativity is lost mainly for larger values of $m_{1}$. Through the behavior of $\eta_{01}^{(4)}$, we could see the mass thresholds which mostly affect the annihilation of dark matter, and these are at the $c, \tau$ and $b$ masses, as well as $m_{1} / 2$ and $m_{1}$. Also, we have seen that for small values of $m_{1}$, very light dark matter is viable, with a mass as small as $1 \mathrm{GeV}$. This is of course useful for understanding the results of the experiments DAMA/LIBRA, CoGeNT, CRESST Seidel (2010) as well as the recent data of the Fermi Gamma Ray Space Telescope.

The next step was to analyze dark-matter direct detection in the context of this model. We have imposed systematically the relic-density constraint and, in addition, restricted the dark-matter mass regions to be consistent with perturbativity $\left(\eta_{01}^{(4)} \leq 1\right)$. We have found that the model survives current experimental bounds for a wide range of the parameter space, while at the same time recongnizing that most of the allowed mass regions will be probed by the XENON1T experiment.

The last step was to use an example to see how low-energy phenomenology can restrain the paramaters' space. We have analysed the decay of the meson $B_{S}$ into a pair of $\mu^{+} \mu^{-}$and saw how this could constrain significantly the $S_{1}$ mass and the $S_{1}$ - Higgs mixing angle $\theta$. Other processes can be envisaged, and further constraints should be expected (abada \& Nasri, 2011). Implications on the Higgs detection through the measurable channels should also be considered as current experimental bounds from LEP II data can be used to constrain the mixing angle $\theta$ and possibly other parameters.

This model can be investigated in other directions. For example, the $S_{1}$ vacuum expectation value $v_{1}$ was taken equal to $100 \mathrm{GeV}$, but a priori, nothing prevents us from considering other scales. However, taking $v_{1}$ much larger than the electro-weak scale requires $\eta_{01}^{(4)}$ to be very small, which will result in the suppression of the crucial annihilation channel $S_{0} S_{0} \rightarrow S_{1} S_{1}$. Also, we have fixed the Higgs mass to $m_{h}=138 \mathrm{GeV}$, which is consistent with the current acceptable experimental bounds (Nakamura et al., 2010). Yet, it can be useful to ask here too what the effect of changing this mass would be. Finally, in this study, besides the dark matter field $S_{0}$, only one extra field has been considered. Naturally, one can generalize the investigation to include $N$ such fields and discuss the cosmology and particle phenomenology in terms of $N$. It just happens that the model is rich enough to open new possibilities in the quest for dark matter worth pursuing. At the same time, it tells us that modeling cold dark matter is as challenging as it is exciting.

\section{References}

Zwicky, T.; Helv. Phys. Acta 6, 124 (1933).

Persic, M., Salucci, P., Stel, F.; Mon. Not. Roy. Astron. Soc. 281, 27 (1996) (arXiv:astro-ph/9506004).

Fabricant, D., Gorenstein, P.; Ap. J. 267 (1983) 535.

Stewart, G.C., Canizares, C.R., Fabian, A.C., and Nilsen, P.E.J.; Ap. J. 278 (1984) 53.

Mellier, Y.; Ann. Rev. Ast. Astr. 37 (1999) 127.

Spergel, D.N. et al. [WMAP Collaboration]; Astrophys. J. Suppl. 170 (2007) 377 [arXiv:astro-ph/0603449].

Pope, A. et al. [The SDSS Collaboration]; Astrophys. J. 607 (2004) 655 (arXiv:astro-ph/0401249).

Steigman, G.; arXiv:1008.4765 [astro-ph.co]. 
Komatsu. E. et al.; arXiv: 1001.4538 [astro-ph.Co].

Amsler, C., et al. [Particle Data Group]; Phys. Lett. B 667, 1 (2008).

Javorsek, D. et al.; Phys. Rev. Lett. 87, 231804 (2001).

Javorsek, D. et al.; Phys. Rev. D 65, 072003 (2002).

Ellis, J., Hagelin, J., Nanopoulos, D., Olive, K. and Srednicki, M.; Nucl. Phys. B238 (1984) 453.

Jungman, G., Kamionkowski, M. and Griest, K.; Phys. Rept. 267 (1996) 195 (arXiv: hep-ph/9506380).

Belanger, G., Boudjema, F., Pukhov, A. and Singh, R.; JHEP 0911, 026 (2009).

Akrami, Y., Scott, P., Edsjo, J., Conrad, J. and Bergstrom, L.; JHEP 1004, 057 (2010).

Vasquez, D., Belanger, G., Boehm, C., Pukhov, A.and Silk, J.; Phys. Rev. D 82, 115027 (2010).

Feldman, D., Liu, Z. and Nath, P.; Phys. Rev. D81 (2010) 117701 (arXiv:1003.0437 [hep-ph]).

Kuflik, E., Pierce, A. and Zurek, K.; Phys. Rev. D81 (2010) 111701.

Fornengo, N., Scopel, S. and Bottino, A.; Phys. Rev. D 83, 015001 (2011).

Silveira, V. and Zee, A.; Phys. Lett. B161 (1985) 136.

McDonald, J.; Phys. Rev. D50 (1994) 3637.

Burgess, C., Pospelov, M. and ter Veldhuis, T.; Nucl. Phys. B619 (2001) 709.

Barger, V., Langacker, P., McCaskey, M., Ramsey-Musolf, M. and Shaughnessy, G.; Phys. Rev. D77 (2008) 035005 (arXiv: 0706.4311 [hep-ph]).

Gonderinger, M., Li, Y., Patel, H. and Ramsey-Musolf, M.; JHEP 053 (2010) 1001, 2010 (arXiv:0910.3167 [hep-ph]).

He, X., Li, T., Li, X., Tandean, J. and Tsai, H.; Phys. Rev. D79 (2009) 023521 (arXiv : 0811.0658 [hep-ph]).

Asano, M. and Kitano, R.; Phys. Rev. D 81, 054506 (2010).

Angle, J. et al. [XENON Collaboration]; Phys. Rev. Lett. 100 (2008) 021303 (arXiv : 0706.0039 [astro-ph]).

Ahmed, Z. et al. [CDMS Collaboration]; Phys. Rev. Lett. 102 (2009) 011301 (arXiv:0802.3530 [astro-ph]).

Arina, C. and Tytgat, M.; JCAP 1101, 011 (2011).

Abada, A., Nasri. S. and Ghaffor, D.; Phys. Rev. D83, 095021 (2011).

Aprile, E. et al. [XENON100 Collaboration]; Phys. Rev. Lett. 105 (2010) 131302 (arXiv:1005.0380 [astro-ph.CO]).

Schnee, R. et al. [The SuperCDMS Collaboration]; arXiv: astro-ph/ 0502435.

Aprile, E. et al. [Xenon Collaboration]; J. Phys. Conf. Ser. 203 (2010) 012005.

Nakamura, K. et al. [Particle Data Group]; J. Phys. G37 (2010) 075021.

The effect of $\eta_{0}$ in the one-real-scalar extension of the Standard Model is discussed in Spergel, D. and Steinhardt, P.; Phys. Rev. Lett. 84 (2000) 3760 (astro-ph/9909386).

Kolb, E. and Turner, M.; 'The Early Universe', Addison-Wesley, (1998).

Bernabei, R., Belli, P., Cappella, F. et al. [DAMA/LIBRA Collaboration]; Eur. Phys. J. C67 (2010) 39 (arXiv:1002.1028 [astro-ph.GA]).

Aalseth, C.E. et al. [CoGeNT Collaboration]; arXiv:1002.4703 [astro-ph.CO] .

Gaitskell, R., Mandic, V. and Filippini, J.; SUSY Dark Matter/Interactive Direct Detection Limit Plotter; http: / / dmtools. berkeley. edu/limitplots.

Abada, A. and Nasri, S.; work in progress.

Seidel, W.; WONDER 2010 Workshop, Laboratory Nazionali del Gran Sasso, Italy, March 22-23, 2010; IDM 2010 Workshop, Montpellier, France, July 26-30, 2010. 


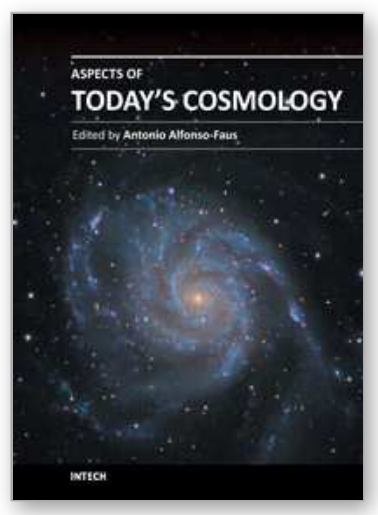

\author{
Aspects of Today's Cosmology \\ Edited by Prof. Antonio Alfonso-Faus
}

ISBN 978-953-307-626-3

Hard cover, 396 pages

Publisher InTech

Published online 09, September, 2011

Published in print edition September, 2011

This book presents some aspects of the cosmological scientific odyssey that started last century. The chapters vary with different particular works, giving a versatile picture. It is the result of the work of many scientists in the field of cosmology, in accordance with their expertise and particular interests. Is a collection of different research papers produced by important scientists in the field of cosmology. A sample of the great deal of efforts made by the scientific community, trying to understand our universe. And it has many challenging subjects, like the possible doomsday to be confirmed by the next decade of experimentation. May be we are now half way in the life of the universe. Many more challenging subjects are not present here: they will be the result of further future work. Among them, we have the possibility of cyclic universes, and the evidence for the existence of a previous universe.

\title{
How to reference
}

In order to correctly reference this scholarly work, feel free to copy and paste the following:

Abdessamad Abada and Salah Nasri (2011). Modeling Light Cold Dark Matter, Aspects of Today's Cosmology, Prof. Antonio Alfonso-Faus (Ed.), ISBN: 978-953-307-626-3, InTech, Available from: http://www.intechopen.com/books/aspects-of-today-s-cosmology/modeling-light-cold-dark-matter

\section{INTECH}

open science | open minds

\section{InTech Europe}

University Campus STeP Ri

Slavka Krautzeka 83/A

51000 Rijeka, Croatia

Phone: +385 (51) 770447

Fax: +385 (51) 686166

www.intechopen.com

\section{InTech China}

Unit 405, Office Block, Hotel Equatorial Shanghai

No.65, Yan An Road (West), Shanghai, 200040, China 中国上海市延安西路65号上海国际贵都大饭店办公楼 405 单元

Phone: +86-21-62489820

Fax: $+86-21-62489821$ 
(C) 2011 The Author(s). Licensee IntechOpen. This chapter is distributed under the terms of the Creative Commons Attribution-NonCommercialShareAlike-3.0 License, which permits use, distribution and reproduction for non-commercial purposes, provided the original is properly cited and derivative works building on this content are distributed under the same license. 